\title{
Non-Similar Solution for Magnetized Flow of Maxwell Nanofluid over an Exponentially Stretching Surface
}

\author{
Raheela Razzaq, ${ }^{1}$ Umer Farooq, ${ }^{1}$ Jifeng Cui, ${ }^{2}$ and Taseer Muhammad $\mathbb{D}^{3,4}$ \\ ${ }^{1}$ Department of Mathematics, COMSATS University Islamabad, Islamabad Campus, Chak Shehzad, Islamabad 44000, Pakistan \\ ${ }^{2}$ College of Science, Inner Mongolia University of Technology, Hohhot 010051, China \\ ${ }^{3}$ Department of Mathematics, College of Sciences, King Khalid University, Abha 61413, Saudi Arabia \\ ${ }^{4}$ Mathematical Modelling and Applied Computation Research Group (MMAC), Department of Mathematics, \\ King Abdulaziz University, P. O. Box 80203, Jeddah 21589, Saudi Arabia
}

Correspondence should be addressed to Taseer Muhammad; taseer_qau@yahoo.com

Received 3 March 2021; Revised 5 April 2021; Accepted 17 May 2021; Published 28 May 2021

Academic Editor: Carlos Llopis-Albert

Copyright ( 92021 Raheela Razzaq et al. This is an open access article distributed under the Creative Commons Attribution License, which permits unrestricted use, distribution, and reproduction in any medium, provided the original work is properly cited.

In this study, an analysis is made by studying more reliable nonsimilar magneto-hydrodynamics (MHD) flow of Maxwell fluid with nanomaterials. Nonsimilar transport is produced by extending of sheet with arbitrary velocity. Maxwell structure is marked to indicate the non-Newtonian fluid behavior. The leading nondimensional partial differential system (PDEs) is transmuted to a set of the nonlinear ordinary differential system (ODEs) through local nonsimilarity technique. The developing system is solved numerically using an implemented package known as bvp4c in MATLAB. The analysis discovers several physical features of thermal and velocity profiles. Remark the flow accelerated for greater Deborah and Hartman parameters. The influence of thermophoresis number on the thermal figure is minimal. The conducts of velocity, concentration, and thermal distribution and local Nusselt number and skin friction are illustrated graphically by taking distinct parameters. The consequences disclose that the local Nusselt number is an increasing function of Prandtl number; however, it is a decaying function for Brownian motion. The rise in skin friction is observed for increasing Brownian motion and Lewis numbers.

\section{Introduction}

Many of the recent technological implementations involving standard fluids are now developed through insertion of fibers of nanometer-sized particles to primary fluids, so-called nanofluids. One of the main utilization of nanofluids is to enhance the heat shifting performance of base fluid. As the time passes, development and advancement in the technology are made due to nanotechnology, and it will have a huge impact in future market. Nanotechnology is defined as the technological appliances based on the study of structures at the nanoscale level. The recent developments in nanotechnology [1] have created effective products. Nanotechnology is used for the production of medicine, therapy, diagnostic and sequencing, electronics, medicines, and manufacturing materials.

The investigations on MHD flows [2-5] and rate of heat shifting are judged as of intense concern owing to the consequences of magnetic field in various products utilizing electrically conducting fluids. Few engineering implementations of MHD are energy generators, gas turbines polymer mechanization, geothermal energy extractions, plasma studies, cooling of nuclear reactors, crystal growth, and metallurgy. Magnetic nanofluid carried both the liquid and magnetic features. Various physical features of such liquids are adapted by deviating the magnetic field. Additionally, magnetized nanoliquids are helpful in the manufacturing and biomedicine applications. Biomaterial for wound treatment, sterilized devices, and gastric medications are examples. The magnetized nanoparticles can be utilized in eradicating tumors with hyperthermia for magnetic resonance imaging and targeted drug release. Hayat et al. [6] exhibited impacts of convective conditions on MHD nanoliquid flow through porous sheet above surface expanding at an exponential rate. Turkyilmazoglu [7] 
explored three-dimensional MHD flow and heat transfer above a stretching/shrinking surface in a viscoelastic fluid.

Recently, the studies about non-Newtonian fluids bring about huge interest because of its numerous usages in industrial products. Mathematically, such kind of fluids is unable to describe through one basic equation. Thus, distinct constitutive equations are suggested in perspective diversity of such fluids. The simplest non-Newtonian rate-type fluid is the Maxwell fluid model that can impressively characterize the antecedent of the relaxation time effect. Due to its relatively simple structure, it can recruit various problems. In past, Fetecau et al. [8] acquired exact solution for flow of Maxwell fluid. Maxwell fluid flow in porous medium is studied by Wang and Hayat [9]. Fetecau and Fetecau [10] presented exact solutions of Maxwell fluid for unsteady flow. A two-dimensional MHD Maxwell fluid is studied by Hayat et al. [11]. Heyhat and Khabazi [12] explored MHD Maxwell fluid flow with convective top over a plan stiff zone.

Recently, a remarkable progress has been done in the field of nanofluid over stretching surfaces. This new field is drawing attention of many researchers to make a significant progress. Fluid motions because of the expanding surfaces are very important as it has many applications in the manufacturing industry which include manufacturing of polymers and metal sheets, material handling conveyers, liquid film along the boundary layer, and the plastic sheets used in the aerodynamic expulsion. Crane [13] inaugurated the study of 2D viscous liquid flow with linearly expanding velocity using similarity transformations. Liao [14] illustrated nonsimilar boundary layer flow of viscous fluid restricted to an expanding sheet. You and $\mathrm{Xu}$ [15] supplied nonsimilar outcomes for transport of second level liquid above an expanding surface along random velocity. Kousar and Liao [16] exhibited nonsimilar free convective flow of viscous fluid above a permeable sheet. Nakhchi et al. [17] provided analysis for the nonsimilar model of thermal layer flow above an expanding flat plate. Kousar et al. [18] addressed nonsimilarity boundary layer flow of viscous fluid on a porous wedge. Cui et al. [19] exhibited nonsimilar flow for mixed convective flows of viscous fluids with nanoparticles.

Boundary layer flows describing the significance of noslip condition were proposed by Prandtl [20]. The boundary layer momentum and continuity equations were first solved by a student of Prandtl, a German engineer named Blasius [21]. It was concluded by transforming two PDE's into one ODE by launching a new independent variable, familiar as similarity variable. In this technique, the independent variables emerging in PDE should be one less than the primary equations. ODE's acquired after transformation can be linear or nonlinear. The linear system of ODE's can be resolved easily as compared to the nonlinear system of ODE's. Numerical and analytical techniques are commonly operated to assess these ODE's. Analytical methods are mostly utilized to resolve similar flows cause of its calculation and ideal modesty. Sometimes, it may happen that even after applying transformations, the system is not fully transformed into ODE's because of the presence of independent variable in the physical parameters and last in the shape of PDE which can be more accurately described through nonsimilar equations.

Most of real world applications are nonsimilar in nature, which is an innovative point of this article. Nonsimilar boundary layer flows are more important not only theoretically but also have vast applications in real life. Despite of this fact, there are fewer publications in the field of nonsimilar flows as compared to similar flows. Farooq et al. [22] provided analytical series solution of nonsimilar boundary layer flow.

In this research method, local nonsimilarity for $2^{\text {nd }}$ level of truncation via bvp4c is employed to get the solutions. In the method of local similarity ( $1^{\text {st }}$ level truncation), PDE's obtained after transformation have similar and nonsimilar terms. Assuming the nonsimilarity terms of the equation very small and ignoring these expressions, the PDE's becomes ODE's [23]. The key imperfection of the method of local similarity is neglecting the nonsimilar terms. The outcomes calculated by the procedure of local similarity were not accurate. To overcome this drawback, Minkowycz and Sparrow [24] presented a new method called "Method of local nonsimilarity ( $2^{\text {nd }}$ level truncation)" for obtaining solution with nonsimilar terms which are not zero. Later, nonsimilar flows were studied by Massoudi [25].

Our aim is to propose nonsimilarity transformations which then develop the nonsimilar model of Maxwell nanofluid. In this procedure, the physical coordinates $(x, y)$ and are transformed to $(\xi, \eta)$ dimensionless coordinates. If $\xi$ is zero or a constant, then the nonsimilar problem is converted into similar problem. In regard of mentioned debate, MHD fluid flow with nanomaterial above exponential expanding sheet is demonstrated in this article. The PDE's are changed into ODE's which can be more conveniently approximated as compared to the original PDE. The bvp4c MATLAB-based algorithm will be used to compute coupled highly nonlinear boundary value problems. The quantities of interest local Sherwood and Nusselt numbers, profiles of velocity, temperature, and concentration are inspected by graphs and tables.

\section{Mathematical Formulation}

A 2D MHD laminar, incompressible transport of Maxwell nanofluid over an exponentially expanding surface along velocity and $U_{I}$, concentration $C_{I}$, and temperature $T_{I}$ on the wall is shown in Figure 1 by making use of boundary layer theory as exposed as follows:

$$
\begin{aligned}
\frac{\partial u}{\partial x}+\frac{\partial v}{\partial y}= & 0 \\
u \frac{\partial u}{\partial x}+v \frac{\partial v}{\partial y}= & v\left(\frac{\partial^{2} u}{\partial y^{2}}\right)-\lambda\left(u^{2} \frac{\partial^{2} u}{\partial x^{2}}+2 u v \frac{\partial^{2} u}{\partial x \partial y}+v^{2} \frac{\partial^{2} u}{\partial y^{2}}\right) \\
& -\frac{\sigma B_{0}^{2}}{\rho_{f}}\left(\lambda v \frac{\partial u}{\partial y}+u\right) \\
u \frac{\partial T}{\partial x}+v \frac{\partial T}{\partial y}= & \alpha\left(\frac{\partial^{2} T}{\partial y^{2}}\right)+\tau D_{B}\left(\frac{\partial C}{\partial y} \frac{\partial T}{\partial y}\right)+\frac{\tau D_{T}}{T_{\infty}}\left(\frac{\partial T}{\partial y}\right)^{2}
\end{aligned}
$$




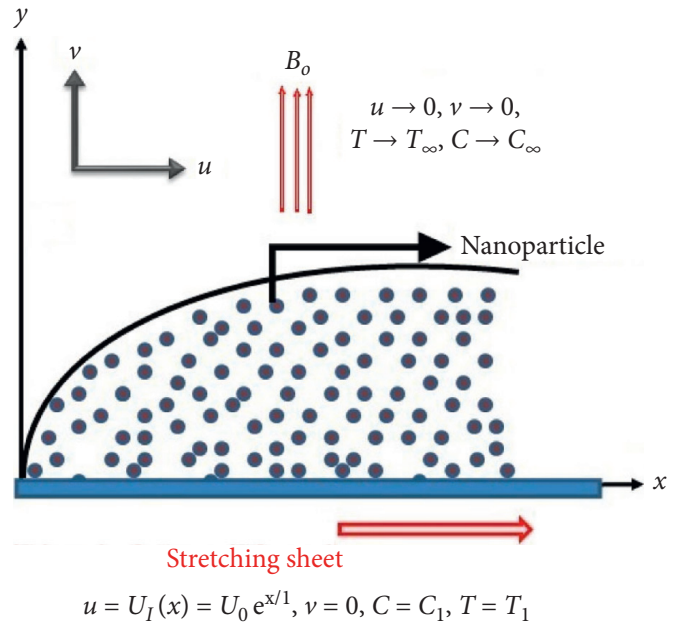

Figure 1: Physical configuration.

$$
u \frac{\partial C}{\partial x}+v \frac{\partial C}{\partial y}=D_{B}\left(\frac{\partial^{2} C}{\partial y^{2}}\right)+\frac{D_{T}}{T_{\infty}}\left(\frac{\partial^{2} T}{\partial y^{2}}\right)
$$

In this model, nanoparticles' heat capacity ratio to original heat capacity is $\tau$, the acceleration cause of gravity is $g$, the Brownian diffusion coefficient is $D_{B}$ and, the kinematic viscosity is $\mu$, specific heat is $C_{p}$, and coefficient of thermophoresis diffusion is $D_{T} ; C_{\infty}$ and $T_{\infty}$ are the free stream concentration and temperature, respectively, $C$ is the volume fraction of the nanoparticles, $T$ is the temperature, $\lambda$ is relaxation time, $\alpha$ describes the thermal diffusivity, and $\rho_{f}$ is the fluid density.

The boundary conditions are

$$
\left\{\begin{array}{l}
u=U_{I}(x)=U_{0} \exp \frac{x}{l} \\
v=0 \\
C=C_{I}=C_{\infty}+C_{0} \exp \left(\frac{x}{2 l}\right) \\
T=T_{I}=T_{\infty}+T_{0} \exp \left(\frac{x}{2 l}\right), \\
\text { at } y=0
\end{array}\right.
$$

$$
\begin{aligned}
& \text { As } y \longrightarrow \infty \text {, } \\
& v \longrightarrow 0 \text {, } \\
& u \longrightarrow 0 \text {, } \\
& T \longrightarrow T_{\infty} \text {, } \\
& C \longrightarrow C_{\infty} \text {. }
\end{aligned}
$$

The nonsimilar flow is developed by introducing new parameters $\xi$ and $\eta$ denominated as nonsimilarity variable and pseudosimilarity variable.

$$
\begin{aligned}
& \xi=\exp \left(\frac{x}{l}\right), \\
& \eta=y \sqrt{\frac{U_{0}}{2 v l} \exp \left(\frac{x}{2 l}\right) .}
\end{aligned}
$$

Now, by using chain rule for connecting the parameters in $(x$, and $y)$ plane to those in $(\xi, \eta)$ plane, we get

$$
\left\{\begin{array}{l}
u=U_{0} \exp \left(\frac{x}{l}\right) \frac{\partial f}{\partial \eta}(\xi, \eta) \\
v=-\sqrt{\frac{v U_{0}}{2 l} \exp \left(\frac{x}{2 l}\right)\left(f(\xi, \eta)+\eta \frac{\partial f}{\partial \eta}+2 \xi \frac{\partial f}{\partial \xi}\right)} \\
T=\theta(\xi, \eta)\left(T_{I}-T_{\infty}\right)+T_{\infty} \\
C=\phi(\xi, \eta)\left(C_{I}-C_{\infty}\right)+C_{\infty} .
\end{array}\right.
$$

Substitution of (8) in (1)-(6), we get the following dimensionless nonsimilar system:

$$
\begin{aligned}
\frac{\partial^{2} f}{\partial \eta^{3}}+ & f \frac{\partial^{2} f}{\partial \eta^{2}}-2\left(\frac{\partial f}{\partial \eta}\right)^{2}-2 M^{2} \frac{\partial f}{\partial \eta} \xi^{-1} \\
& +\beta\left(M^{2} f \frac{\partial^{2} f}{\partial \eta^{2}}+M^{2} \eta \frac{\partial f}{\partial \eta} \frac{\partial^{2} f}{\partial \eta^{2}}-2 \xi\left(\frac{\partial f}{\partial \eta}\right)^{3}\right. \\
& +\frac{1}{2} \eta \xi\left(\frac{\partial f}{\partial \eta}\right)^{2} \frac{\partial^{2} f}{\partial \eta^{2}}-6 \xi^{2}\left(\frac{\partial f}{\partial \eta}\right)^{2} \frac{\partial^{2} f}{\partial \xi}-2 \xi^{3}\left(\frac{\partial f}{\partial \eta}\right)^{2} \frac{\partial^{3} f}{\partial \xi^{2} \partial \eta} \\
& +3 \xi f \frac{\partial f}{\partial \eta} \frac{\partial^{2} f}{\partial \eta^{2}}+2 \xi^{2} f \frac{\partial^{2} f}{\partial \xi} \frac{\partial f}{\partial \eta^{2}}+6 \xi^{2} \frac{\partial f}{\partial \eta} \frac{\partial f}{\partial \eta} \frac{\partial^{2} f}{\partial \eta^{2}} \\
& +4 \xi^{3} \frac{\partial f}{\partial \eta} \frac{\partial f}{\partial \xi} \frac{\partial^{3} f}{\partial \xi}-\frac{1}{2} \xi f^{2} \frac{\partial^{3} f}{\partial \eta^{3}}-2 \xi^{2} f \frac{\partial^{2} f}{\partial \eta^{2} \partial \xi} \\
= & 2 \xi\left(\frac{\partial f}{\partial \eta} \frac{\partial^{2} f}{\partial \xi}-\frac{\partial^{2} f}{\partial \eta^{2}} \frac{\partial f}{\partial \xi}\right), \\
& \left.-2 \xi^{2} \frac{\partial^{2} f}{\partial \eta^{2}}\left(\frac{\partial f}{\partial \xi}\right)^{2}+2 \xi M^{2} \frac{\partial^{2} f}{\partial \eta^{2}} \frac{\partial f}{\partial \xi}\right)
\end{aligned}
$$

$$
\begin{aligned}
\frac{\partial^{2} \theta}{\partial \eta^{2}} & +\operatorname{Pr}\left(f \frac{\partial \theta}{\partial \eta}-\theta \frac{\partial f}{\partial \eta}+\xi\left(N_{b} \frac{\partial \theta}{\partial \eta} \frac{\partial \xi}{\partial \eta}+N_{t}\left(\frac{\partial \theta}{\partial \eta}\right)^{2}\right)\right) \\
= & 2 \operatorname{Pr} \xi\left(\frac{\partial f}{\partial \eta} \frac{\partial \theta}{\partial \xi}-\frac{\partial \theta}{\partial \eta} \frac{\partial f}{\partial \xi}\right),
\end{aligned}
$$

$\frac{\partial^{2} \phi}{\partial \eta^{2}}+\frac{N_{t}}{N_{b}} \frac{\partial^{2} \theta}{\partial \eta^{2}}+S c\left(f \frac{\partial \phi}{\partial \eta}-\frac{\partial f}{\partial \eta} \phi\right)=2 \xi S c\left(\frac{\partial f}{\partial \eta} \frac{\partial \phi}{\partial \xi}-\frac{\partial \phi}{\partial \eta} \frac{\partial f}{\partial \xi}\right)$

In the above dimensionless nonsimilar equations, $M$ is the Hartman number (ratio of electromagnetic force to the 
viscous force), $\beta$ is the Deborah number (ratio of relaxation time to the observation/experimental time), $N_{t}$ is the thermophoresis (a force generated due to change in temperature between the hot gas/fluid and the cold surface effecting the particulate movement towards the cold surface), Pr is the Prandtl number (ratio of kinematic viscosity to thermal diffusivity), $N_{b}$ is the Brownian motion (is a result of the collisions of the particles with other fast accelerating particles in the fluid), and $S c$ (ratio of momentum diffusivity/ kinematic viscosity and mass diffusivity) is defined as

$$
\begin{aligned}
\beta & =\frac{\lambda U_{o}}{l}, \\
\operatorname{Pr} & =\frac{v}{\alpha}, \\
M^{2} & =\frac{\sigma l B_{o}^{2}}{\rho_{f} U_{o}}, \\
N_{b} & =\frac{\tau D_{B} C_{0}}{v}, \\
N_{t} & =\frac{\tau T_{o} D_{T}}{v T_{\infty}}, \\
\text { Le } \operatorname{Pr} & =S c .
\end{aligned}
$$

Nonsimilar boundary conditions are

$$
\begin{aligned}
\frac{\partial f}{\partial \eta}(\xi, 0) & =1, \\
\phi(\xi, \infty) & =0, \\
\phi(\xi, 0) & =1, \\
f(\xi, 0)+2 \xi \frac{\partial f}{\partial \xi} & =0, \\
\frac{\partial f}{\partial \eta}(\xi, \infty) & =0, \\
\theta(\xi, \infty) & =0, \\
\theta(\xi, 0) & =1 .
\end{aligned}
$$

The parameters Ec, $\operatorname{Re}_{x}, N_{b}, M$, Le, and Pr represent the Eckert number, Reynolds number, Brownian motion, thermophoresis, magnetic field, Lewis number, and Prandtl number, respectively.

The local Sherwood number Sh, skin friction coefficient $C_{f}$, and the local Nusselt number $\mathrm{Nu}$ are

$$
\begin{aligned}
\mathrm{Nu} & =\frac{x q_{I}}{k\left(T_{1}-T_{\infty}\right)}, \\
C_{f} & =\frac{2 \tau_{I}}{\rho U_{I}^{2}}, \\
\mathrm{Sh} & =\frac{\mathrm{xj}_{I}}{D_{B}\left(C_{I}-C_{\infty}\right)},
\end{aligned}
$$

where

$$
\begin{aligned}
\tau_{I} & =\mu\left(\frac{\partial u}{\partial y}\right)_{y=0}, \\
j_{I} & =-D_{B}\left(\frac{\partial C}{\partial y}\right)_{y=0}, \\
q_{I} & =-k\left(\frac{\partial r}{\partial y}\right)_{y=0}, \\
(2 \operatorname{Re})^{(1 / 2)} C_{f} & =\sqrt{2} f^{\prime \prime}(\xi, 0), \\
\operatorname{Re}^{(-1 / 2)} \mathrm{Sh} & =-\sqrt{\frac{1}{2}} X \phi^{\prime}(\xi, 0), \\
\operatorname{Re}^{(-1 / 2)} \mathrm{Nu} & =-\sqrt{\frac{1}{2}} X \phi^{\prime}(\xi, 0) .
\end{aligned}
$$

where $X=\ln \xi$.

\section{Local Nonsimilarity Method}

Local similarity is a frequently used technique in the solution of nonsimilarity boundary layers. In this opinion, term and $\partial(.) / \partial \xi$ of equations (9)-(11) are deduced to be sufficiently small; therefore, it can be estimated to zero, presume structure of ODE's, and the computational assignment is clarified in (17)-(19). Thus, the local similarity solution or first extent of truncation is computationally appealing, but conduct numerical outcomes have doubtful precision. It is because of unsure or ignorance of right edge of equations. To overcome such complications, Sparrow et al. [26] and Sparrow and $\mathrm{Yu}$ [27] awarded local nonsimilarity technique to acquire the outcomes for the nonsimilar boundary layer equations.

3.1. First Level of Truncation. We have neglected the terms on right side of equations (9)-(11) assuming $\xi$ and $(\partial(.) / \partial \xi)$ as small as compared to other terms. We are going to write the ordinary derivatives of functions with respect to $\eta$ and using prime notation and get the system of equations (17)-(19) mentioned:

$$
\begin{aligned}
f^{\prime \prime \prime} & +f^{\prime \prime} f-2 f^{2}-2 M^{2} f^{\prime} \xi^{-1} \\
& +\beta\left(M^{2} f f^{\prime \prime}+M^{2} \eta f^{\prime} f^{\prime \prime}-2 f^{\prime 3} \xi\right. \\
& \left.+\frac{1}{2} \xi \eta f^{\prime 2} f^{\prime \prime}+3 \xi f f^{\prime} f^{\prime \prime}-\frac{1}{2} \xi f^{2} f^{\prime \prime \prime}\right)=0, \\
\theta^{\prime \prime} & +\operatorname{Pr}\left(f \theta^{\prime}-f^{\prime} \theta+\xi^{(1 / 2)}\left(N_{b} \theta^{\prime} \phi^{\prime}+N_{t} \theta^{2}\right)\right)=0, \\
\phi^{\prime \prime} & +\frac{N_{t}}{N_{b}} \theta^{\prime \prime}+S c\left(f \phi^{\prime}-f^{\prime} \phi\right)=0 .
\end{aligned}
$$

Boundary conditions are 


$$
\begin{aligned}
f(\xi, 0) & =0, \\
f^{\prime}(\xi, 0) & =1, \\
\phi(\xi, 0) & =1, \\
\phi(\xi, \infty) & =0, \\
f^{\prime}(\xi, \infty) & =0, \\
\theta(\xi, \infty) & =0, \\
\theta(\xi, 0) & =1 .
\end{aligned}
$$

Equations (16)-(18) present a formation of ordinary differential equations (ODE's).
3.2. Second Level of Truncation. Establishing new functions to acquire the larger extent of iteration,

$$
\begin{aligned}
& g(\xi, \eta)=\frac{\partial f(\xi, \eta)}{\partial \xi} \\
& q(\xi, \eta)=\frac{\partial \theta(\xi, \eta)}{\partial \xi} \\
& p(\xi, \eta)=\frac{\partial \phi(\xi, \eta)}{\partial \xi} .
\end{aligned}
$$

Now, by using (21) in (9)-(11), our equations are transformed as

$$
\begin{aligned}
f^{\prime \prime \prime} & +f^{\prime \prime} f-2 f^{\prime 2}-2 M^{2} f^{\prime} \xi^{-1}+\beta\left(M^{2} f f^{\prime \prime}+M^{2} \eta f^{\prime} f^{\prime \prime}-2 \xi f^{3}+\frac{1}{2} \eta \xi f^{\prime 2} f^{\prime \prime}+3 \xi f f^{\prime} f^{\prime \prime}\right. \\
= & \frac{1}{2} \xi f^{2} f^{\prime \prime \prime}-6 \xi^{2} f^{\prime 2} g^{\prime}-2 \xi^{3} f^{\prime 2} g^{\prime 2}-2 \xi^{2} f f^{\prime} g^{\prime \prime}+6 \xi^{2} f^{\prime} f^{\prime \prime} g+4 \xi^{3} f^{\prime} g^{\prime \prime} g-2 \xi^{3} f^{\prime \prime \prime} g^{2}+2 \xi^{2} f f^{\prime \prime \prime} g+2 \xi M^{2} g f^{\prime \prime} \\
= & 2 \xi\left(f^{\prime} g^{\prime}-f^{\prime \prime} g\right), \\
\theta^{\prime \prime}+\operatorname{Pr}\left(\theta^{\prime} f-\theta f^{\prime}+\xi^{(1 / 2)}\left(N_{b} \theta^{\prime} \phi^{\prime}+N_{t} \theta^{\prime 2}\right)\right) & =2 \operatorname{Pr} \xi\left(f^{\prime} q-\theta^{\prime} g\right) \\
\phi^{\prime \prime}+\frac{N_{t}}{N_{b}} \theta^{\prime \prime}+S c\left(f \phi^{\prime}-f^{\prime} \phi\right) & =2 S c \xi\left(f^{\prime} p-\theta^{\prime} g\right) .
\end{aligned}
$$

The boundary conditions are

$$
\begin{aligned}
f^{\prime}(\xi, 0) & =1, \\
f^{\prime}(\xi, \infty) & =0, \\
f(\xi, 0)+2 \xi g(\xi, 0) & =0, \\
\theta(\xi, \infty) & =0, \\
\theta(\xi, 0) & =1, \\
\phi(\xi, \infty) & =0, \\
\phi(\xi, 0) & =1 .
\end{aligned}
$$

We get equations (26)-(28) by taking partial derivatives of (22)-(25) with respect to $\xi$ :

$$
\begin{gathered}
g^{\prime \prime \prime}+3 f^{\prime \prime} g+g^{\prime \prime} f-6 f^{\prime} g^{\prime}-2 M^{2} g^{\prime} \xi^{-1} g^{\prime}-2 M^{2} g^{\prime} \xi^{-2}-2 \xi\left(g^{\prime 2}-g^{\prime \prime} g\right) \\
+\beta\left(M^{2}\left(3 f^{\prime \prime} g+g^{\prime \prime} f+\eta f^{\prime \prime} g^{\prime}+\eta g^{\prime \prime} f^{\prime}+\xi g g^{\prime \prime}-12 \xi f^{\prime 2} g^{\prime}\right)\right. \\
+9 \xi f^{\prime} f^{\prime \prime} g+7 \xi f f^{\prime} g^{\prime \prime}+\eta \xi f^{\prime} f^{\prime \prime} g^{\prime}+\frac{1}{2} \eta \xi f^{\prime 2} g^{\prime \prime}+12 \xi^{2} f^{\prime} g g^{\prime \prime} \\
-4 \xi^{3} f^{\prime} g^{\prime 3}-6 \xi^{2} f^{\prime 2} g^{\prime 2}+6 \xi^{2} g f^{\prime \prime} g^{\prime}+2 \xi^{2} g^{\prime \prime} f g^{\prime}+4 \xi^{3} g^{\prime \prime} g g^{\prime}-3 \xi f f^{\prime \prime \prime} g \\
-8 \xi^{2} f^{\prime \prime \prime} g^{2}-\frac{1}{2} f^{2} f^{\prime \prime \prime}-\frac{1}{2} \xi f^{2} g^{\prime \prime \prime}-2 \xi^{2} f g^{\prime \prime \prime}-2 \xi^{2} g^{2} g^{\prime \prime \prime}=0, \\
q^{\prime \prime}+\operatorname{Pr}\left(\left(q^{\prime} f-3 q f^{\prime}+3 \theta^{\prime} g-g^{\prime} \theta\right)-\frac{1}{2} \xi^{(-1 / 2)}\left(N_{b} \theta^{\prime} \phi^{\prime}+N_{t} \theta^{\prime 2}\right)-\xi^{(1 / 2)}\left(N_{b}\left(p^{\prime} \theta^{\prime}+\phi^{\prime} q^{\prime}\right)+2 N_{t} \theta^{\prime} q^{\prime}\right)-2 \operatorname{Pr} \xi\left(g^{\prime} q-q^{\prime} g\right)=0,\right. \\
(27) \\
p^{\prime \prime}+\frac{N_{t}}{N_{b}} q^{\prime \prime}+S c\left(3 g \phi^{\prime}+p^{\prime} f-3 f^{\prime} p-g^{\prime} \phi\right)-2 S c \xi\left(g^{\prime} p-p^{\prime} g\right)=0 .
\end{gathered}
$$


Boundary conditions are

$$
\begin{aligned}
g(\xi, 0) & =0, \\
g^{\prime}(\xi, 0) & =0, \\
p(\xi, 0) & =0, \\
p(\xi, \infty) & =0, \\
g^{\prime}(\xi, \infty) & =0, \\
q(\xi, 0) & =0, \\
q(\xi, \infty) & =0 .
\end{aligned}
$$

The expressions $(\partial g(\xi, \eta) / \partial \xi),(\partial q(\xi, \eta) / \partial \xi)$, and $(\partial p$ $(\xi, \eta) / \partial \xi)$ and its derivatives were omitted regarding and $\eta$, at this stage of truncation to get accurate data which were first explored by Chen [28]. Farooq et al. [23] successfully used the local nonsimilarity method.

\section{Results and Discussion}

Numerical solutions have been calculated by using the MATLAB-based algorithm bvp4c. Graphs have been obtained for various values of and $M, N_{t}$, and $N_{b}$, and Sc, Hartman number, thermophoresis parameter, Brownian motion, and Schmidt number, for nondimensional temperature, velocity, and concentration profiles.

Figures 2 and 3 show the influences of $\beta$ and for distinct $M$ on velocity profile. It is exhibited that liquid transport is greatest in ambient liquid for slight $\beta$. Although, liquid qualities varies from Newtonian to the non-Newtonian attribute for larger Deborah number; thus, the flow expresses descending trait. Layer thickness deduces as we enlarge $M$ and and $\beta$. Figure 4 shows the evaluation of temperature, for higher value of Brownian motion $N_{b}$ and the number of nanoparticles increases, due to which the surface temperature is enhanced. Figure 5 shows varying values of $\operatorname{Pr}$ on $\theta(\eta)$ at fixed values of physical parameters. The $\operatorname{Pr}$ enhanced because of relatively less thermal diffusivity and the lesser conduction decline the thermal thickness. So, temperature is weaker function of $\mathrm{Pr}$. It is expressed in boundary layer region as temperature reduces with Pr. Analysis of Figure 6 temperature profile for a sheet expanding with $U_{I}$ velocity for variations of $\xi$ discloses that greater $\xi$ reduction in thermal configuration is explored when $\eta \longrightarrow$ (and; then, temperature profile asymptotically approaches to zero. At leading corner, temperature of fluid is identical to surface temperature and discounted to temperature of neighboring fluid. The effect of increase in $\xi$ is to enhance the temperature in the nearby fluid. Figure 7 shows the concentration $\phi(\eta)$ for various values of $N_{t}$ (thermophoresis). An extraordinary decay is observed in the vicinity of exponentially expanding sheet for greater values of $N_{t}$; then, expansion in concentration is noticed. Figure 8 shows the progress of concentration $\phi(\eta)$ with transverse coordinate $\eta$ for diversity of $N_{b}$ Brownian motion parameter. A rise in temperature explored close to the wall and temperature vanishes as $\eta \longrightarrow \infty$. Higher value of $N_{b}$ exhibits the existence of larger values of nanoparticles which are responsible for expansion in surface temperature. The Brownian motion is delicate for larger

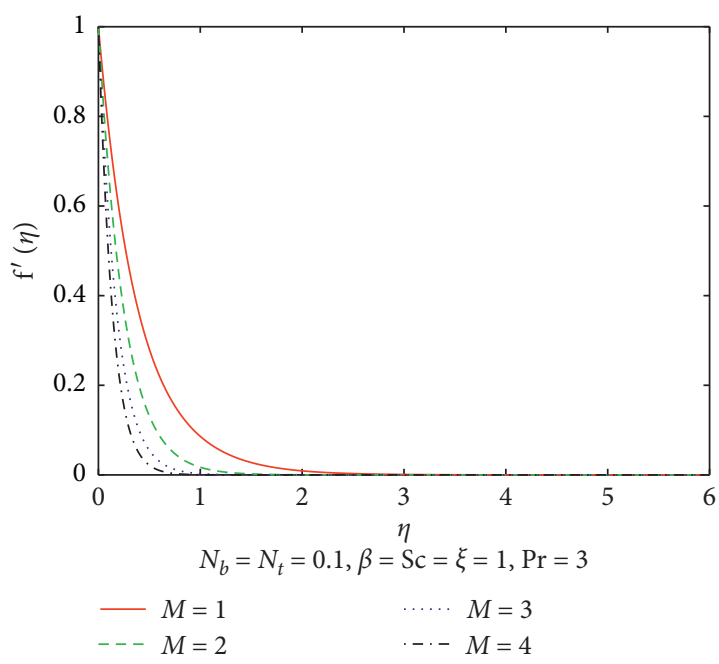

FIGURE 2: Graph of $f^{\prime}(\eta)$ for marked $M$.

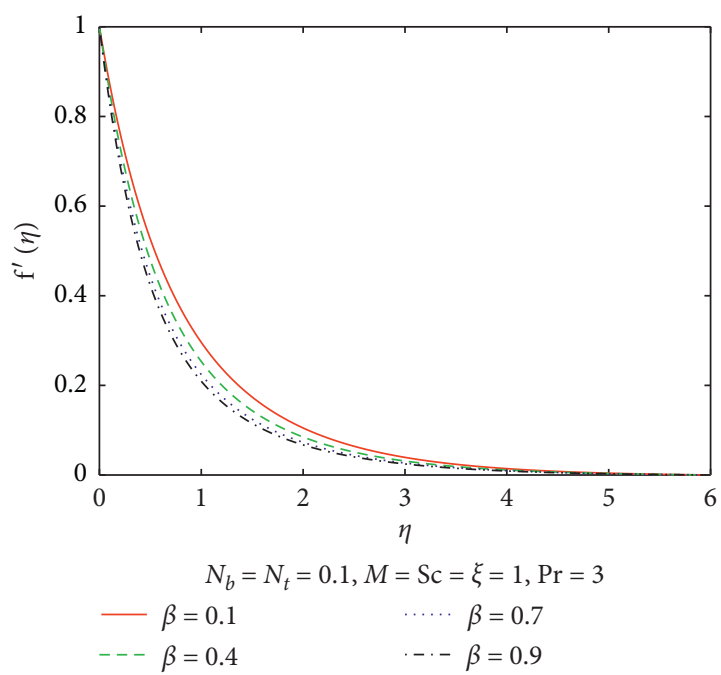

Figure 3: Graph of $f^{\prime}(\eta)$ for different $\beta$.

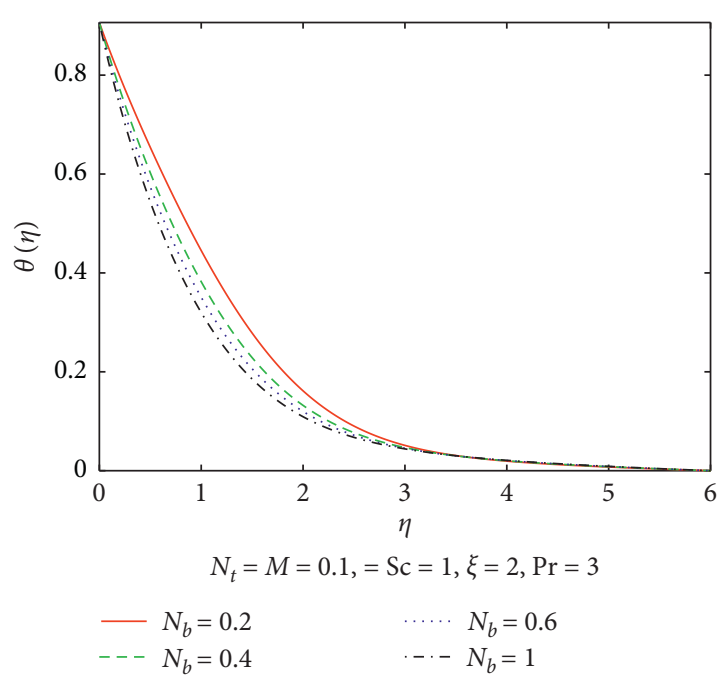

Figure 4: Graph of $\theta(\eta)$ for marked $N_{b}$. 


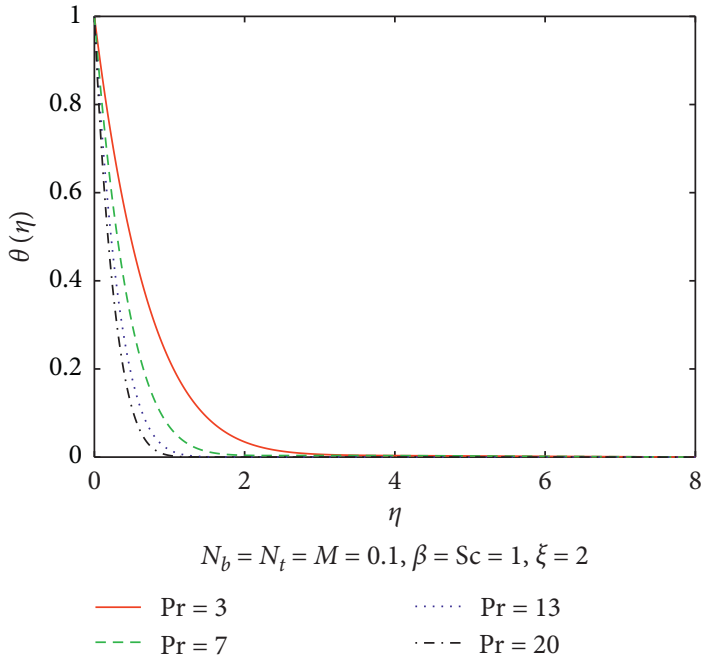

Figure 5: Graph of $\theta(\eta)$ for marked $P r$.

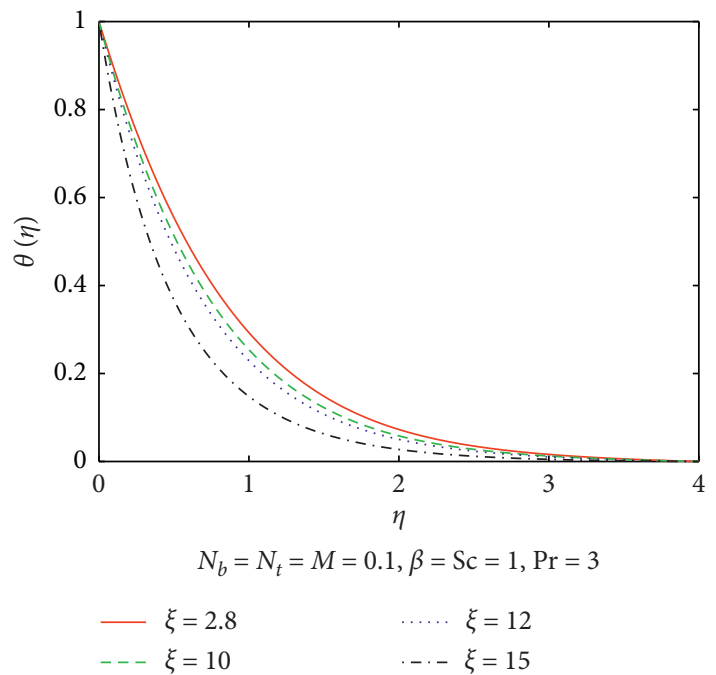

Figure 6: Graph of $\theta(\eta)$ for marked $\xi$.

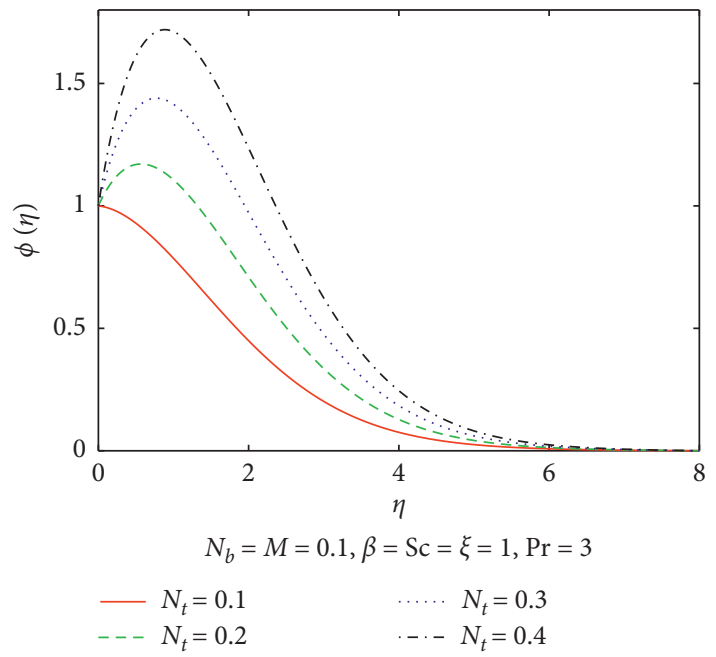

Figure 7: Graph of $\phi(\eta)$ for marked $N_{t}$.

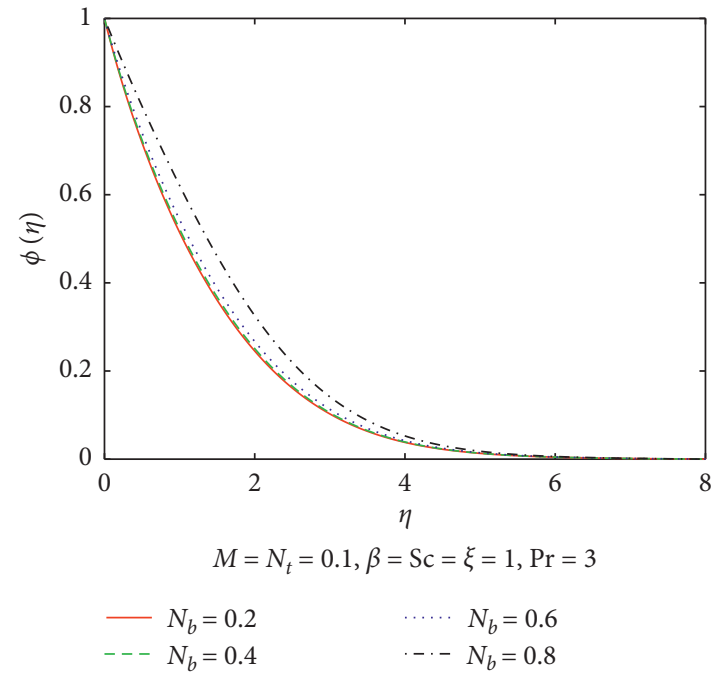

Figure 8: Graph of $\theta(\eta)$ for marked $N_{b}$.

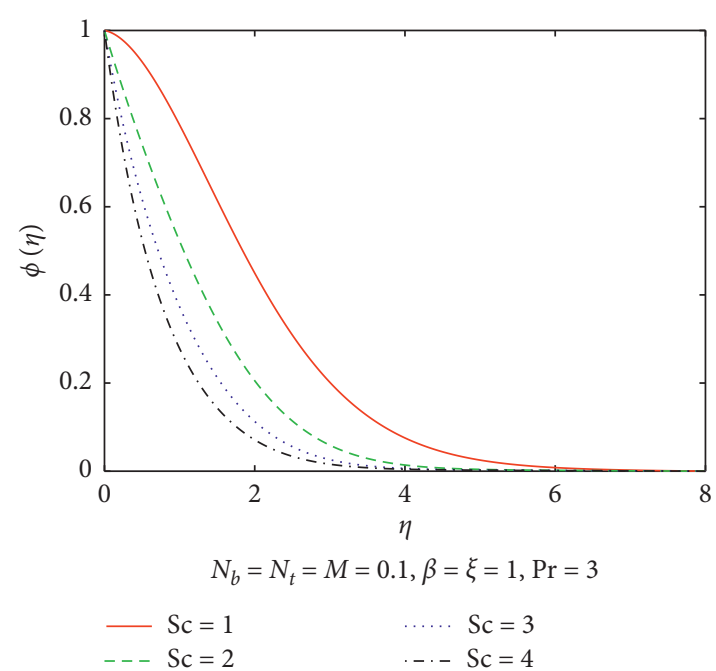

FIgURE 9: Graph of $\phi(\eta)$ for marked Sc.

nanoparticles for smaller $N_{b}$, and for a small-sized nanoparticle, strong Brownian motion is observed. The concentration of the nanoparticles elevated the thermal conductivity of the base fluid. Figure 9 exhibits the influences of Scand on the nanoparticles concentration, and for larger value of and $\mathrm{Sc}$, and $\phi(\eta)$ is decreasing. Schmidt number is compound of Lewis and Prandtl number. The rise in Lewis number exhibited a broad thermal diffusivity that is a response to low heat capacity and higher thermal conductivity.

Table 1 presents the expansion in local skin friction as enhancing values of $\beta$ and. Table 2 describes that Sherwood number decreases with variations in Pr. Table 3 presents that the local Nusselt number expands as the value of $N_{b}$ increases. Table 4 presents the comparison of present outcomes with Mustafa et al. [29] and reduced Nusselt number shown variation in Le and Pr. 
TABLE 1: $C_{f x}$ (local skin friction) for different values of $\beta$ and with comparison of $M$ when $N_{b}=N_{t}=0.1, \operatorname{Pr}=2, \xi=1$, and $S c=1$.

\begin{tabular}{ccc}
\hline$\beta$ & $M$ & $C_{\mathrm{fx}}\left(\mathrm{Re}_{x}\right)^{(1 / 2)}$ \\
\hline \multirow{3}{*}{1} & 1 & -2.4400759418 \\
& 2 & -3.7488981642 \\
& 3 & -5.2321466320 \\
\hline \multirow{3}{*}{3} & 1 & -2.8322827303 \\
& 2 & -4.2454338241 \\
& 3 & -5.8754332961 \\
\hline
\end{tabular}

TABLE 2: Sh (local Sherwood number) for different variations of Sc and $N_{t}$ when $N_{b}=M=0.1, \beta=1$, and $\xi=1$, and $\operatorname{Pr}=2$.

\begin{tabular}{lcc}
\hline Sc & $N_{t}$ & $\operatorname{Sh}\left(\operatorname{Re}_{x}\right)^{(1 / 2)}$ \\
\hline \multirow{3}{*}{1} & 0.2 & 0.8199647873 \\
& 0.4 & 2.3190229815 \\
& 0.6 & 5.4823876138 \\
\hline \multirow{2}{*}{2} & 0.2 & -0.0292898466 \\
& 0.4 & 1.0998928934 \\
& 0.6 & 2.0440720335 \\
\hline & 0.2 & -0.6150890477 \\
& 0.4 & 0.3141505321 \\
& 0.6 & 1.0653271044 \\
\hline
\end{tabular}

TABLe 3: $\mathrm{Nu}_{x}$ (local Nusselt number) for different values of $\xi$ and and $\operatorname{Pr}$ when $N_{t}=N_{b}=M=0.1$ and Sc $=\beta=1$.

\begin{tabular}{ccc}
\hline$\xi$ & $\mathrm{Pr}$ & $\mathrm{Nu}_{x}\left(\mathrm{Re}_{x}\right)^{(-1 / 2)}$ \\
\hline \multirow{3}{*}{1} & 7 & -2.4319244196 \\
& 13 & -3.2308808400 \\
& 20 & -3.9322233752 \\
\hline & 7 & -2.1780753769 \\
& 13 & -2.9108212623 \\
3 & 20 & -3.5450112732 \\
\hline & 7 & -1.1939905457 \\
& 13 & -2.5987902122 \\
& 20 & -2.7617202153 \\
\hline
\end{tabular}

TABLE 4: A comparison for reduced Nusselt number is presented for variations of $\mathrm{Pr}$, Le when $N_{t}=N_{b}=0.1, M=0$, $\beta=0.2$, and and $\xi=1$.

\begin{tabular}{ccccc}
\hline \multicolumn{5}{c}{$-\mathrm{Nu}_{x}\left(\mathrm{Re}_{x}\right)^{(-1 / 2)}$} \\
Pr & Le & Mustafa et al. [29] & Present & $\begin{array}{c}\text { Difference } \\
\text { (percentage) }\end{array}$ \\
\hline 0.4 & & 0.74994 & 0.5083598506 & 47.52 \\
0.7 & 1 & 1.03430 & 0.7170304970 & 44.24 \\
1.0 & & 1.26020 & 0.8882006092 & 41.88 \\
1.2 & & 1.390720 & 0.9855229887 & 41.11 \\
\hline & 0.4 & 1.28090 & 2.3170466904 & 44.71 \\
1 & 0.7 & 1.26860 & 2.0791647255 & 38.98 \\
& 1.2 & 1.25590 & 1.8711778150 & 32.88 \\
& 1.5 & 1.25050 & 1.7929969996 & 30.25 \\
\hline
\end{tabular}

\section{Conclusions}

In this article, an analysis is made by studying more reliable nonsimilar magneto hydrodynamics (MHD) flow of Maxwell fluid with nanomaterials. Nonsimilar transport is produced by extending of sheet with arbitrary velocity. Maxwell structure is marked to indicate the non-Newtonian fluid behavior. The leading nondimensional partial differential system (PDEs) is transmuted to ODEs through a local nonsimilarity technique [30]. The developing system is solved numerically using an implemented package known as bvp4c in MATLAB. Enhancement in $\beta$ and $M$ enhances flow. Temperature becomes enlarge through expansion in $\beta$ and $M$, whereas it reduces due to growth in $N_{t}$ and $\operatorname{Pr}$. The rise in $\beta, M$, and $N_{t}$ expedite in the concentration account, whereas entirely converse is correct for $N_{b}$. Nusselt number becomes large with a growth in $P r$. Although, local Nusselt number deduces with expanding $\beta, M$ and, and $N_{t}$. Sherwood number becomes a greater function of the $N_{b}$ and and $S c$, while it minimizes for an increment in $N_{t}$.

\section{Abbreviations}

$u$, and $v$ : Velocity component in the direction of $x$ and $y$ $\left(\mathrm{ms}^{-1}\right)$

M: $\quad$ Magnetic field

g: $\quad$ The acceleration cause of gravity $\left(\mathrm{ms}^{-2}\right)$

$T_{I}: \quad$ Prescribed surface temperature and $(K)$

$N u_{x}: \quad$ Local Reynolds number

$T_{o}$ : $\quad$ Reference temperature and $(K)$

$T_{\infty}: \quad$ Variable ambient temperature and $(K)$

$f, \theta, \phi: \quad$ Dependent variables

Le: Lewis number

T: $\quad$ Fluid temperature and $(K)$

$L: \quad$ Reference length and $(m)$

Ec: $\quad$ Eckert number

Sh: Local Sherwood number

Gr: $\quad$ Grashof number

Pr: $\quad$ Prandtl number

$\mathrm{Re}_{x}$ : Mass diffusion coefficient

$D: \quad$ Local Nusselt number

Sc: $\quad$ Schmidt number

$D_{t}$ : Thermophoresis diffusion coefficient

$N_{b}$ : $\quad$ Brownian motion parameter

$D_{B}$ : $\quad$ Brownian diffusion coefficient

$B_{o}: \quad$ Magnetic field parameter $\left(\mathrm{Kmolm}^{-3}\right)$

$C: \quad$ Uniform magnetic field

$C_{o}: \quad$ Reference concentration

$C_{p}: \quad$ Specific heat at constant pressure $\left(K^{-1} s^{-2} m^{2}\right)$

$C_{\infty}$ : The concentration of the ambient fluid

$C_{f}$ : $\quad$ Skin friction coefficient

$U_{o}$ : Reference velocity

$U(x)$ : Stretching velocity

$\mathrm{Nt}: \quad$ Thermophoresis parameter

$\lambda: \quad$ Relaxation time $(s)$

$\mu: \quad$ Dynamic viscosity $(\mathrm{kg} /(\mathrm{ms}))$

$\xi: \quad$ Nonsimilarity variable

$\alpha: \quad$ Thermal diffusivity and $\left(m^{2} s^{-1}\right)$

$\tau$ : $\quad$ Heat capacity 


$\begin{array}{ll}\Psi: & \text { Stream function } \\ \nu: & \text { Kinematic viscosity and }\left(m^{2} s^{-1}\right) \\ \sigma: & \text { The electrical conductivity of the fluid } \\ I: & \text { Condition at the wall } \\ \infty: & \text { Ambient condition } \\ \prime: & \text { Differentiation with respect to } \eta .\end{array}$

\section{Data Availability}

The data used to support the findings of this study are included within the article.

\section{Conflicts of Interest}

The authors declare that they have no conflicts of interest.

\section{Acknowledgments}

The authors extend their appreciation to the Deanship of Scientific Research at King Khalid University, Abha, Saudi Arabia for funding this work through research groups program under grant number R.G.P-1/142/42.

\section{References}

[1] U. Farooq, D. Lu, S. Munir, M. Ramzan, M. Suleman, and S. Hussain, "MHD flow of Maxwell fluid with nanomaterials due to an exponentially stretching surface," Scientific Reports, vol. 9, no. 1, p. 7312, 2019.

[2] K. Jacob, M. S. Wabomba, A. M. Kinyanjui, and M. A. Lunani, "Magnetic field and Hall current effect on MHD free convection flow past a vertical rotating flat plate," Asian Journal of Current Engineering and Maths, vol. 6, pp. 346-354, 2012.

[3] S. Nadeem and R. U. Haq, "Effect of thermal radiation for megnetohydrodynamic boundary layer flow of a nanofluid past a stretching sheet with convective boundary conditions," Journal of Computational and Theoretical Nanoscience, vol. 11, no. 1, pp. 32-40, 2014.

[4] P. Sreenivasulu and N. Reddy, "Thermal radiation and chemical reaction effects on MHD stagnation-point flow of A nanofluid over A porous stretching sheet embedded in A porous medium with heat absorption/generation: lie group Analysis," Journal of Global Research in Mathematical Archives, vol. 1, pp. 13-27, 2013.

[5] M. Turkyilmazoglu, "Multiple solutions of heat and mass transfer of MHD slip flow for the viscoelastic fluid over a stretching sheet," International Journal of Thermal Sciences, vol. 50, no. 11, pp. 2264-2276, 2011.

[6] T. Hayat, T. Muhammad, S. A. Shehzad, G. Q. Chen, and I. A. Abbas, "Interaction of magnetic field in flow of Maxwell nanofluid with convective effect," Journal of Magnetism and Magnetic Materials, vol. 389, pp. 48-55, 2015.

[7] M. Turkyilmazoglu, "Three dimensional MHD flow and heat transfer over a stretching/shrinking surface in a viscoelastic fluid with various physical effects," International Journal of Heat and Mass Transfer, vol. 78, pp. 150-155, 2014.

[8] C. Fetecau, M. Athar, and C. Fetecau, "Unsteady flow of a generalized Maxwell fluid with fractional derivative due to a constantly accelerating plate," Computers \& Mathematics with Applications, vol. 57, no. 4, pp. 596-603, 2009.
[9] Y. Wang and T. Hayat, "Fluctuating flow of a Maxwell fluid past a porous plate with variable suction," Nonlinear Analysis: Real World Applications, vol. 9, no. 4, pp. 1269-1282, 2008.

[10] C. Fetecau and C. Fetecau, "A new exact solution for the flow of a Maxwell fluid past an infinite plate," International Journal of Non-linear Mechanics, vol. 38, no. 3, pp. 423-427, 2003.

[11] T. Hayat, Z. Abbas, and M. Sajid, "MHD stagnation-point flow of an upper-convected Maxwell fluid over a stretching surface," Chaos, Solitons \& Fractals, vol. 39, no. 2, pp. 840-848, 2009.

[12] M. M. Heyhat and N. Khabazi, "Non-isothermal flow of Maxwell fluids above fixed flat plates under the influence of a transverse magnetic field," Proceedings of the Institution of Mechanical Engineers, Part C: Journal of Mechanical Engineering Science, vol. 225, no. 4, pp. 909-916, 2011.

[13] L. J. Crane, "Flow past a stretching plate," Zeitschrift für angewandte Mathematik und Physik ZAMP, vol. 21, no. 4, pp. 645-647, 1970.

[14] S.-J. Liao, "A general approach to get series solution of nonsimilarity boundary-layer flows," Communications in Nonlinear Science and Numerical Simulation, vol. 14, no. 5, pp. 2144-2159, 2009.

[15] X. Y. Cheng and X. Hang, "Non-similarity solution of doublediffusive free convection flow from a vertical surface," AIP Conference Proceedings, vol. 1978, no. 1, 470017 pages, 2018.

[16] N. Kousar and S. Liao, "Series solution of non-similarity natural convection boundary-layer flows over permeable vertical surface," Science China Physics, Mechanics and Astronomy, vol. 53, no. 2, pp. 360-368, 2010.

[17] M. E. Nakhchi, M. R. H. Nobari, and H. B. Tabrizi, "Nonsimilarity thermal boundary layer flow over a stretching flat plate," Chinese Physics Letters, vol. 29, no. 10, Article ID 1047039, 2012.

[18] N. Kousar, R. Mahmood, and A. Nawaz, "Series solutions of nonlinear coupled equations in tissue engineering," Alexandria Engineering Journal, vol. 57, no. 3, pp. 2069-2075, 2018.

[19] J. Cui, U. Farooq, R. Razzaq, W. A. Khan, and M. A. Yousif, "Closure to computational analysis for mixed convective flows of viscous fluids with nanoparticles," Journal of Thermal Science and Engineering Applications, vol. 11, no. 2, Article ID 021013, 2019.

[20] A. J. Ghajar, "Heat and Mass Transfer," 6th Fundamentals \& Applications, p. 944, McGraw-Hill, New York, NY, USA, 2001.

[21] L. Prandtl, "Über Flussigkeitsbewegung bei sehr kleiner Reibung. Verhandl. III," International Mathematical, vol. 1904, pp. 484-491, 1904.

[22] U. Farooq, T. Hayat, A. Alsaedi, and S. J. Liao, "Series solutions of non-similarity boundary layer flows of nano-fluids over stretching surfaces," Numerical Algorithms, vol. 70, no. 1, pp. 43-59, 2015.

[23] U. Farooq, M. A. Khan, M. I. Khan, and S. J. Liao, "Modeling and non-similar Analysis for Darcy-Forcheimer-Brinkman model of Casson fluid in a porous media," International Communications of Heat and Mass Transfer, vol. 119, pp. 0735-1933, 2020.

[24] W. J. Minkowycz and E. M. Sparrow, "Local non-similar solutions for natural convection on a vertical cylinder," Journal of Heat Transfer, vol. 96, 1974.

[25] M. Massoudi, "Local non-similarity solutions for the flow of a non-Newtonian fluid over a wedge," International Journal of Non-linear Mechanics, vol. 36, no. 6, pp. 961-976, 2001.

[26] E. M. Sparrow, H. Quack, and C. J. Boerner, "Local nonsimilarity boundary layer solutions," American Institute of 
Aeronautics and Astronautics Journal, vol. 8, no. 11, pp. 1936-1942, 1970.

[27] E. M. Sparrow and H. S. Yu, "Local non-similarity thermal boundary-layer solutions," Journal of Heat Transfer, vol. 93, no. 4 , pp. $328-334,1971$.

[28] T. .S. Chen, "Parabolic Systems: Local Nonsimilarity Method," Handbook of Numerical Heat Transfer, pp. 183-214, 1988.

[29] M. Mustafa, M. A. Farooq, T. Hayat, and A. Alsaedi, "Numerical and series solutions for stagnation-point flow of nanofluid over an exponentially stretching sheet," PLOS ONE, vol. 8, no. 5, 2013.

[30] U. Farooq, R. Razzaq, M. I. Khan, Y. M. Chu, and D. C. Lu, "Modeling and numerical computation of nonsimilar forced convective flow of viscous material towards an exponential surface," International Journal of Modern Physics B, 2021. 